\title{
From Paris to Glasgow
}

\author{
The catalysis of $\mathrm{CO}_{2}$ conversion is a research topic ripe with potential to contribute towards a net-zero future. \\ This Focus issue features a collection of content dedicated to progressing the fundamental science and practical \\ implementation of this technology to advance climate goals.
}

F ive years ago, the Paris Agreement - the international treaty that limits the maximum allowed rise in average global temperatures to $2{ }^{\circ} \mathrm{C}$ (preferably 1.5 ${ }^{\circ} \mathrm{C}$ ) below pre-industrial levels - came into effect ${ }^{1}$. It was drafted over the course of two weeks in late 2015 in a suburb of Paris at the COP21 meeting of the the United Nations Framework Convention on Climate Change (UNFCCC). This was lauded to be a landmark agreement, which went further than the earlier Kyoto Protocol in establishing a specific limit for temperature. The individual countries involved in the agreement are required to submit their plans for $\mathrm{CO}_{2}$ emissions reduction every five years with the stipulation that ambition must be increased in each iteration.

\section{"The individual countries involved in the agreement are required to submit their plans for $\mathrm{CO}_{2}$ emissions reduction every five years with the stipulation that ambition must be increased in each iteration."}

As we reach the five-year anniversary of the agreement and the first of these global stocktakes, we must acknowledge the bumpy road that has been travelled. The initiative hit a major setback in late 2019 when the United States, one of the world's major polluting countries ${ }^{2}$, announced its intention to withdraw from the accord (even though their eventual exit was only for a period of months before they re-joined). During the same period, the world's attentions have been diverted by the difficulties of the COVID-19 pandemic. Initial hopes that the resulting behavioural changes would lead to a decrease in emissions turned out to be short-lived $^{3}$. In the run-up to the COP26 meeting in Glasgow, it can be difficult not to feel disheartened by the headlines indicating that many of the 192 parties to the accord are not setting targets with the level of ambition required to reach the climate goals ${ }^{4,5}$.
While international politics can be notoriously slow moving, we must look to successes and possibilities in the areas where we can make a tangible contribution. The community of scientists whose dream is to reinvent $\mathrm{CO}_{2}$ from waste pollutant to a chemical resource have been working at pace. In this Focus issue of Nature Catalysis, we present a collection of content dedicated to the conversion of $\mathrm{CO}_{2}$ to fuels and feedstock chemicals through catalytic processes. In particular, the fields of electrocatalytic $\mathrm{CO}_{2}$ reduction and heterogeneous $\mathrm{CO}_{2}$ hydrogenation have made enormous strides over the past decade, while big challenges remain.

In line with the aims of our journal, the collection contains content addressing both the fundamental and applied faces of this community. In a pair of Q\&A articles, we speak to the founders of two emerging $\mathrm{CO}_{2}$ conversion start-ups. Staff Sheehan from NYC-based Air Company, brings us along on the scale-up journey of their $\mathrm{CO}_{2}$-to-alcohol hydrogenation technology which has resulted in the first commercially available $\mathrm{CO}_{2}$-derived vodka. Across the Pond, in Bordeaux, David Wakerley and Sarah Lamaison, whose company Dioxycle is designing $\mathrm{CO}_{2}$ electrolysers for industry, give insight into the lived experience at a fledgling green tech start-up.

The practical considerations of a viable $\mathrm{CO}_{2}$ catalysis process are not only the concern of industrial ventures as they also need to guide and inform fundamental research. Two important, and often overlooked issues for the real-world efficiency of $\mathrm{CO}_{2}$ electrolysis are $\mathrm{CO}_{2}$ supply to the system and the purity of the formed products. In Perspective articles, Haotian Wang and co-workers assess the need for high concentration and purity products without downstream purification to reach a competitive cost scenario, while discussing the recent advances in reactor design using porous solid electrolytes to advance this goal. Chengxiang Xiang, David Vermaas, Harry Atwater and colleagues then discuss the integration of $\mathrm{CO}_{2}$ capture and conversion technology by conceptualizing different coupling configurations and weighing their relative merits.
In another Perspective article, Geoffrey Ozin and colleagues analyse the use of $\mathrm{CO}_{2}$-derived methanol as a means to store intermittent solar energy. The pathway presented involves the intermediate formation of $\mathrm{CO}$-rich syngas from $\mathrm{CO}_{2}$ and renewable (solar) $\mathrm{H}_{2}$ via the reverse water-gas shift reaction. Carbon tax levels and the price of renewable $\mathrm{H}_{2}$ emerge as dominant in determining the economic viability of the system in the near and longer terms, respectively.

One of the intrinsic complications involved in $\mathrm{CO}_{2}$ reduction is the rich chemistry of carbon and the consequent large number of potential products, even if only considering $\mathrm{C}_{1}$ products ( $\mathrm{CO}, \mathrm{CH}_{4}, \mathrm{CH}_{3} \mathrm{OH}, \mathrm{CH}_{2} \mathrm{O}, \mathrm{HCOOH}$ ). With the ability of $\mathrm{C}-\mathrm{C}$ coupling and $\mathrm{C}_{2+}$ products, the possibilities are almost endless. Synthesizing catalysts that have high selectivity for a desired product is very challenging and there is clear need for rational design. Jenny Yang and Hannah Shafaat discuss the fundamental factors dictating selectivity in $\mathrm{CO}_{2}$ reduction in a Perspective article where they leverage the inspiration provided by biological systems that have long been able to effectively activate $\mathrm{CO}_{2}$.

Circling back to the policies that form the underlying framework for scientific advancement, Sarah Marie Jordaan and colleagues provide a Comment on how energy policy must interface with $\mathrm{R} \& \mathrm{D}$ to advance the implementation of $\mathrm{CO}_{2}$ catalysis towards achieving the Paris goals.

At the time of writing, world leaders are making their way to Glasgow for COP26 and we will soon learn the outcome of their discussions. It is our sincere hope that this conference will act as a catalyst for positive change, resetting the global emissions trend onto a more sustainable trajectory. Yet, whatever transpires among politicians, we (as scientists) need to continue to play our part, raise our ambition and work together, across borders and across disciplines ${ }^{6}$, to develop practical solutions to the $\mathrm{CO}_{2}$ problem.

Published online: 18 November 2021 https://doi.org/10.1038/s41929-021-00716-9 


\section{References}

1. The Paris Agreement (United Nations, 2015); https://unfccc.int/ process-and-meetings/the-paris-agreement/the-paris-agreement

2. Which countries are the world's biggest carbon polluters?

(Climatetrade, 2021); https://climatetrade.com/which-countries-

are-the-worlds-biggest-carbon-polluters
3. Tollefson, J. Nature 589, 343 (2021).

4. McGrath, M. Climate change: UN emissions gap report a 'thundering wake-up call' $B B C$ https://www.bbc.com/news/ science-environment-59049770 (26 October 2021).

5. Joly, J. Australia sets climate targets but vows to keep coal mines operating for as long as possible. Euronews.green https://www.euronews.com/green/2021/10/26/australia-sets2050-net-zero-emissions-target-but-wont-give-up-coal (26 October 2021)

6. Research in support of COP26. Nature https://www: nature.com/collections/hfggdbdcee (11 October 2021) 